\title{
Chloroplast and Cell Imaging at Submicron Resolution by Two-Photon Excitation
}

\author{
I.C. Baianu \\ FSHN and NPRE Departments, \\ College of ACES and College of Engineering, \\ University of Illinois at Urbana, \\ 305/350 Burnsides Research Laboratory, \\ Urbana, Illinois 61801, USA \\ Email: ibaianu@illinois.edu
}

$04 / 02 / 2012$

\section{Introduction}

Multi-photon (MPE) NIR excitation of fluorophores--attached as labels to biopolymers like proteins and nucleic acids, or bound at specific biomembrane sites-- is one of the most attractive options in biological applications of laser scanning microscopy. Many of the serious problems encountered in spectroscopic measurements of living tissue, such as photodamage, light scattering and auto-fluorescence, can be reduced or even eliminated. FCS can therefore provide accurate in vivo and in vitro measurements of diffusion rates, "mobility" parameters, molecular concentrations, chemical kinetics, aggregation processes, labeled nucleic acid hybridization kinetics and fluorescence photophysics/ photochemistry. Several photophysical properties of fluorophores that are required for quantitative analysis of FCS in tissues have already been widely reported. Molecular "mobilities" can be measured by FCS over a wide range of characteristic time constants from $\sim 10^{-3}$ to $10^{3} \mathrm{~ms}$. At signal levels comparable to $1 \mathrm{PE}$ confocal microscopy, two-photon excitation (2PE) reduces photobleaching in spatially restricted cellular compartments, thereby preserving the long-term signal-to-noise during data acquisition. Furthermore, 3PE has been reported to eliminate DNA damage and photobleaching problems that may still be present in some 2PE experiments. Whereas both $1 \mathrm{PE}$ and $2 \mathrm{PE}$ alternatives are suitable for intracellular FCS observations on thin biological specimens, 2PE can substantially improve FCS signal quality in turbid samples, such as plant cell suspensions or deep cell layers within tissues.

\section{Two-Photon Imaging Instrumentation}

This section presents submicron resolution imaging $(\sim 0.25 \mu \mathrm{m}$ axial) results that we obtained with two-photon NIR excitation (2PE) of FCS. This preliminary FCS data was obtained in collaboration with ISS Co and Dr. Glenn Fried at the Microscopy Suite of the Beckman Institute for Advanced Science and Technology at UIUC by employing two-photon NIR fluorescence excitation at $780 \mathrm{~nm}$ with a $180 \mathrm{fs}$, Ti: Sapphire pulsed laser, coupled to an FCS Alba ${ }^{\text {TM }}$ spectrometer system (recently designed and manufactured by ISS Co., Urbana, Illinois). The configuration of an Alba ${ }^{\mathrm{TM}}$ spectrometer with an inverted microscope is shown in Figure 1, and its optical path and main system components are represented in Figure 2. 


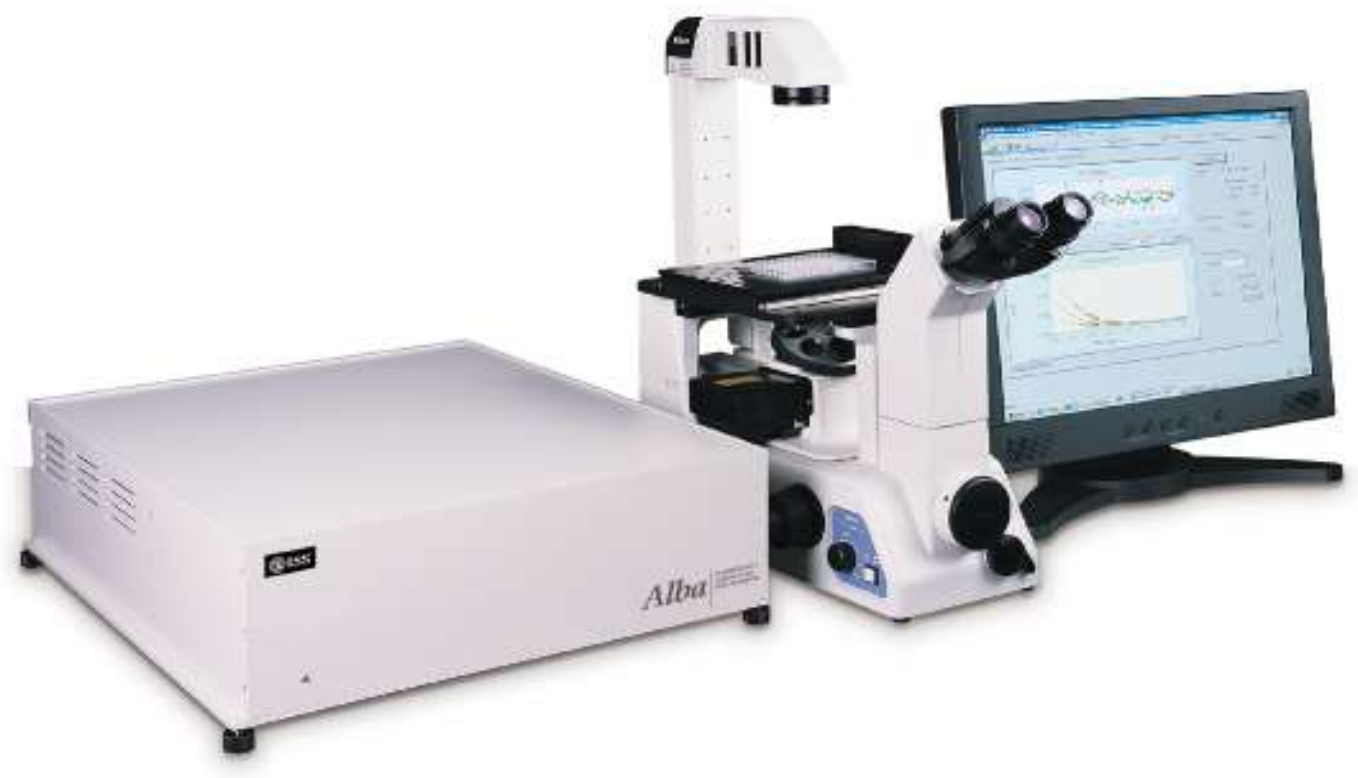

Fig. 1. The FCS Alba ${ }^{\mathrm{TM}}$ Microspectrometer System (manufactured and distributed by the ISS Co., Urbana, Illinois, USA). The inverted, epi-fluorescence microscope shown in the figure is the Nikon TE-300 -special model, that has available both a back illumination port and a lefthand side, $100 \%$ light port. The PC employed for data acquisition, storage and processing is located behind the instrument, as is the laser illumination source (not shown in this figure).

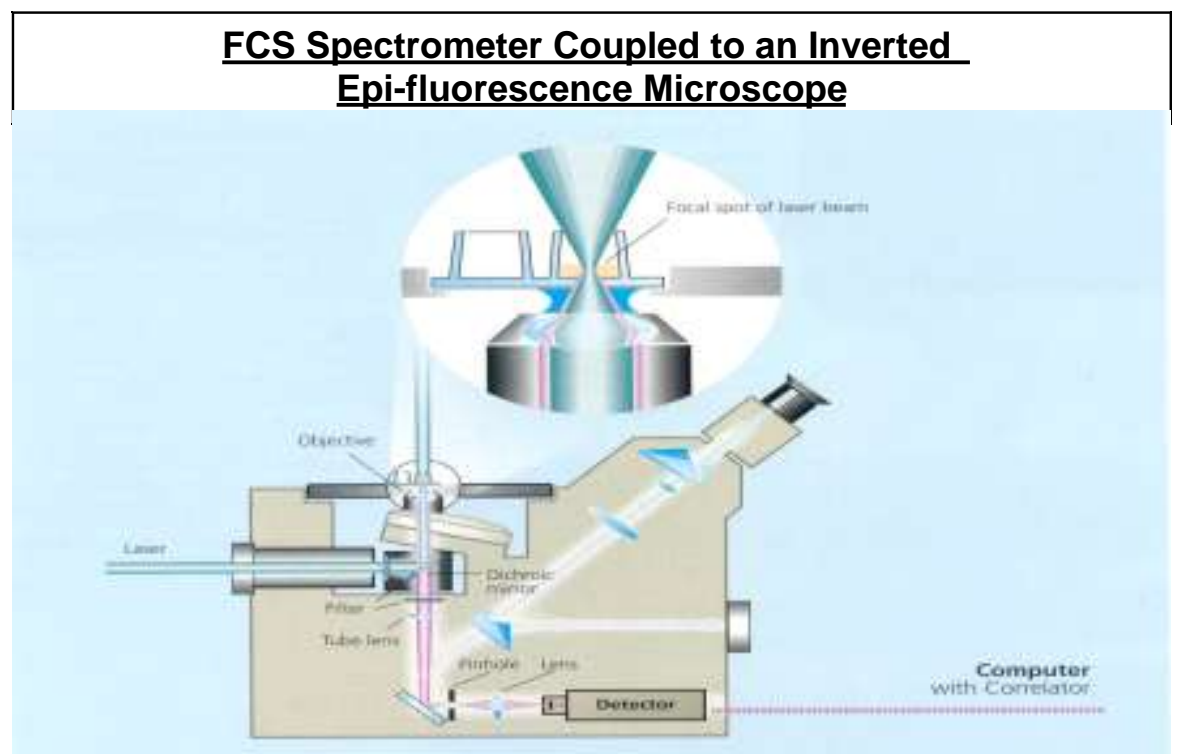

Fig. 2. Diagram of an FCS Spectrometer Coupled to an Inverted Epi-fluorescence Microscope. (Modified from SOURCE: Eigen et al., 1999). 


\section{Experimental Results:}

\section{Single- and Two- Photon Fluorescence Correlation Spectroscopy}

The instrumentation depicted in Figures 1 and 2 allow rapid and very-high sensitivity observations on physiologically functional cells and tissues in vivo.

The results in Figure 3 present single-photon autocorrelation plots obtained with the Alba instrument in Figure 1.

Correlation

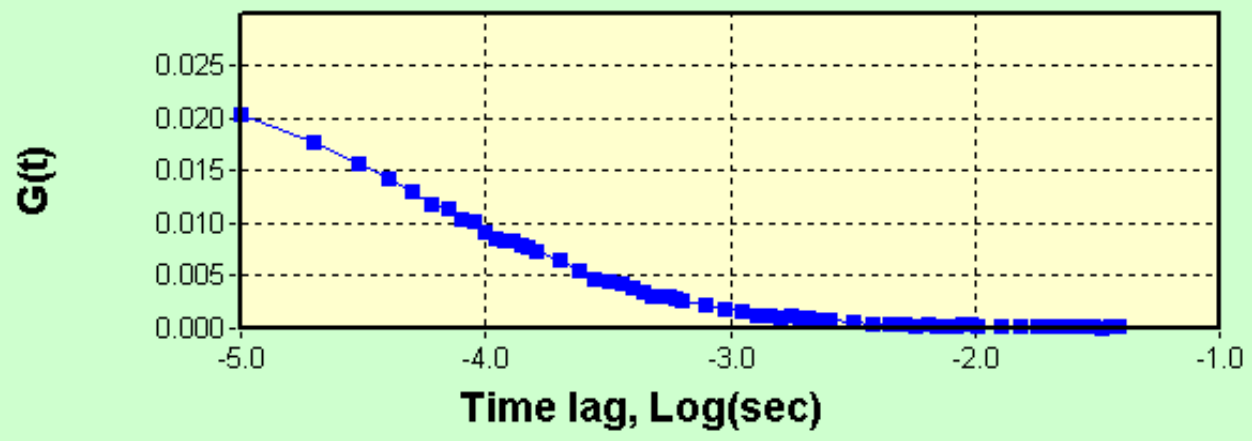

A.

Correlation

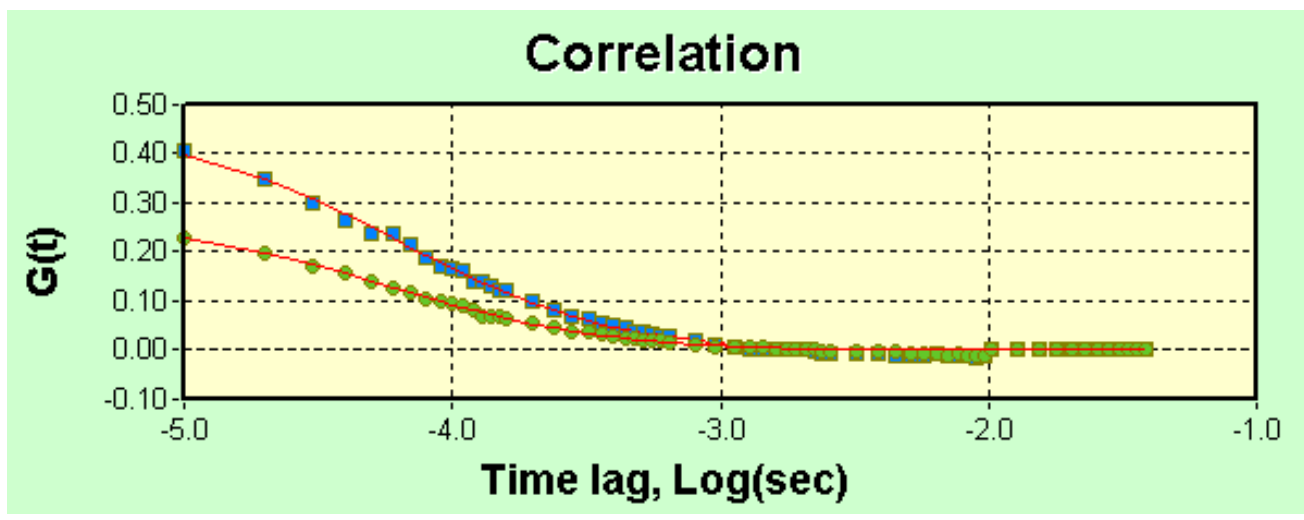

B.

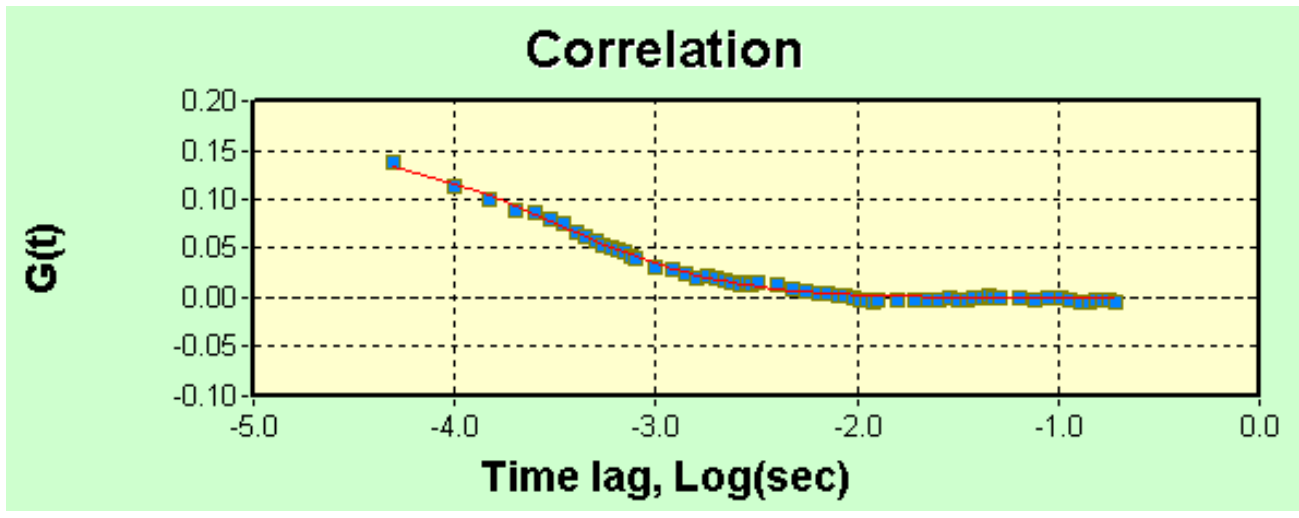

Figure 3. Fitted, Single-Photon, Auto-Correlation Plots:

A. $10 \mathrm{nM}$ Solution of the Fluorescent Dye Rhodamine 110G;

B. 1 and $2 \mathrm{nM}$ Solutions of the Fluorescent Dye Rhodamine 110G obtained with 1PE at $488 \mathrm{~nm}$;

C. FCS Computer Simulation Results. 


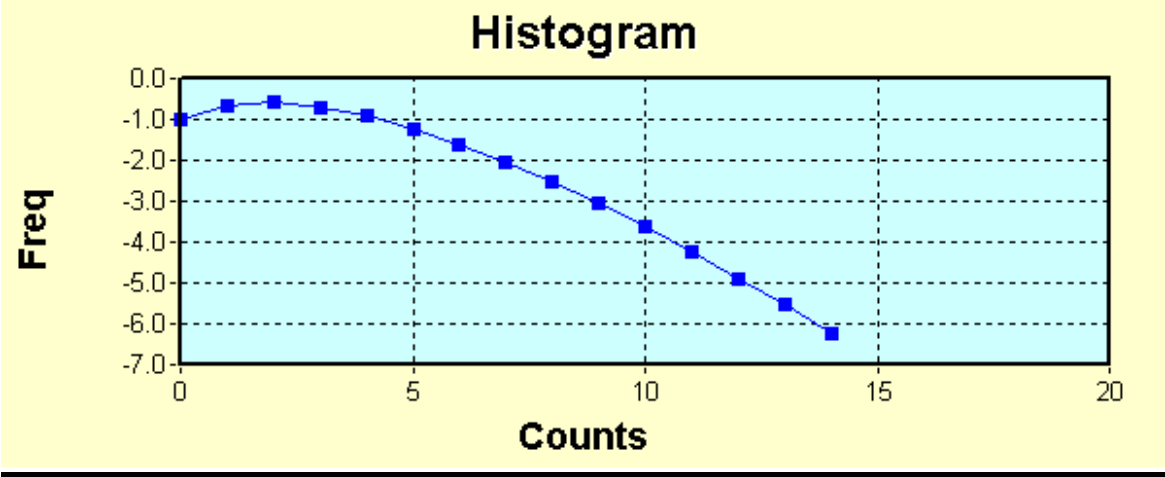

Figure 4. Fluorescence Photon-counting Histogram corresponding to the Correlation in Figure 3A.

The following Figure 5 presents FCS results obtained with two-photon excitation.

A.
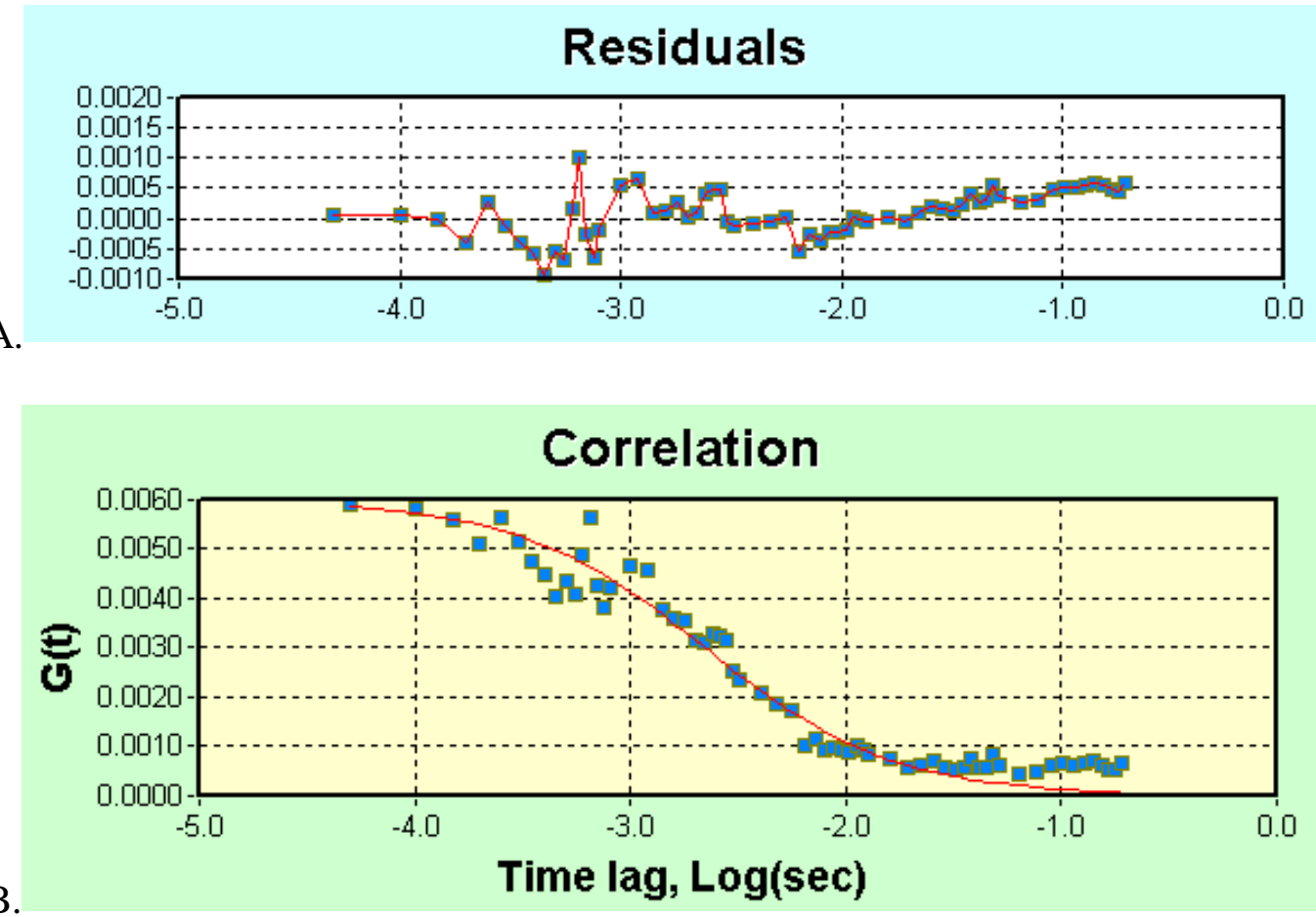

Figure 5. FCS test with two-photon $780 \mathrm{~nm}$ NIR Excitation with 0.25 micron fluorescently labeled spheres. The continuous line shows the regression fitting obtained with the Vista ${ }^{\mathrm{TM}}$ software program developed by ISS Co. (Copyright 2003). 


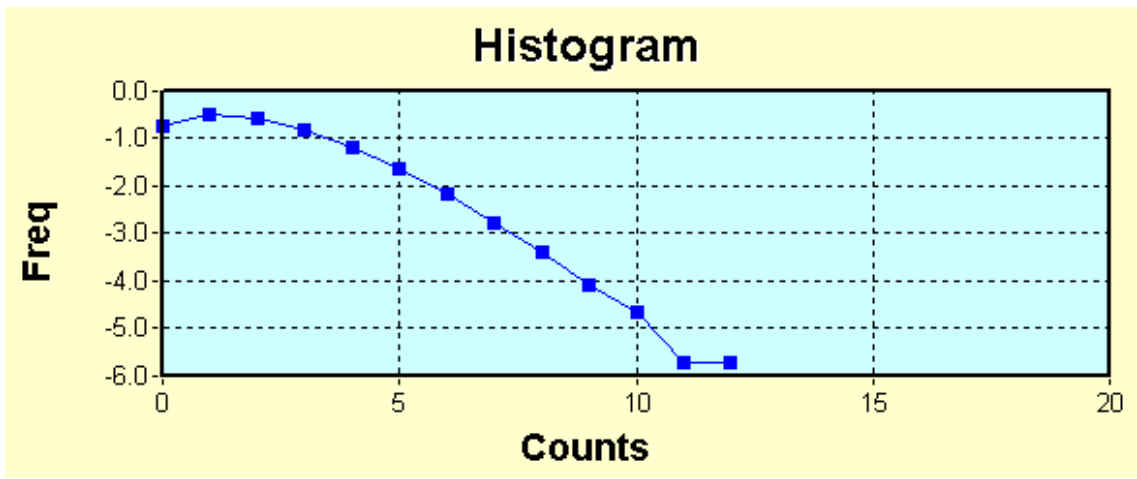

Figure 6. Two-Photon NIR Excitation photon-counting histograms of concentrated (turbid) soybean leaf chloroplast suspensions; (all data were obtained in these tests with 2PE NIR excitation at the $780 \mathrm{~nm}$ NIR wavelength).

\section{Conclusions}

Novel, two-photon NIR excitation fluorescence correlation microspectroscopy tests and preliminary results were presented in this article with submicron resolution for concentrated suspensions of functional cells and chloroplast membranes.

Especially promising are further developments employing multi-photon NIR excitation that could lead, for example, to novel cancer prevention methodology and the early detection of cancers using NIR-excited fluorescence. Other related developments are the applications of Fluorescence Cross-Correlation Spectroscopy (FCCS) detection to monitoring:

DNA- telomerase interactions,

DNA hybridization kinetics,

ligand-receptor interactions, and

HIV-HBV testing.

Very detailed, automated chemical analyses of biomolecules in cell cultures are now also becoming possible by FT-NIR microspectroscopy of single cells, both in vitro and in vivo.

Such rapid analyses have potentially important applications in cancer research, pharmacology and clinical diagnosis. 


\section{References}

1. I.C. Baianu et al. 2004a. High-Resolution Nuclear Magnetic Resonance and Near Infrared Determination of Soybean Oil, Protein and Amino Acid Residues in Soybean Seeds. Ch.11 in $94^{\text {th }}$ AOCS Symp. Proceed., Kansas, MO (2003). AOCS: Champaign (2004).

2. I.C. Baianu et al. 2004b. Near Infrared Microspectroscopy, Fluorescence Microspectroscopy, Infrared Chemical Imaging and High Resolution Nuclear Magnetic Resonance Analysis of Soybean Seeds, Somatic Embryos and Single Cells. Ch.12 in $94^{\text {th }}$ AOCS Symp. Proceed., Kansas, MO (2003). AOCS: Champaign (2004).

3. Schwille P., Oehlenschläger F. and Walter N. (1996) Analysis of RNA-DNA hybridization kinetics by fluorescence correlation spectroscopy, Biochemistry 35, 10182.

4. Chirico, G., Bettati, S., Mozzarelli, A., Chen, Y., Müller, J. D., and Gratton, E. (2001). Molecular Heterogeneity of O-Acetylserine Sulfhydrylase by Two-Photon Excited Fluorescence Fluctuation Spectroscopy. Biophys J, 80(4), 1973-1985.

5. Bagatolli, L.A., and Gratton, E. (2000) Two-photon fluorescence microscopy of coexisting lipid domains in giant unilamellar vesicles of binary phospholipid mixtures. Biophys J. 78, 290-305. 